\title{
Robust outage prevention model for an Unmanned Ariel Vehicle on a predefined area
}

\author{
Cleophas D K Mutepfe ${ }^{1}$, Elisha C Mabunda ${ }^{2}$, Golden P Kapungu ${ }^{3}$ \\ 1,2,3 (University of Zimbabwe, Electrical Engineering Department).
}

\begin{abstract}
This article looks at a remotely operated Unmanned Ariel Vehicles (UAVs) being deployed on a predefined area. A UAV has a communication link for it to be controlled and commanded from the ground. We need to ensure that even under adverse physical and electromagnetic conditions this link is always definable and has an acceptable level of quality of service. This link has to be robust to take into effect Radio Frequency (RF) propagation conditions, interference, line of sight obstruction among other factors. Thus given that a UAV is assigned to do a certain task, this article proposes a solution that guarantees communication between the deployed UAV and the control center even under adverse physical and electromagnetic conditions. A solution of using a number of relay UAVs on a predefined area is presented and a computer model that addresses the issues of topology in which the UAV and relays are deployed, the power budget, geometry and parameters that guarantee redundancy of the system is also presented. This computer model was done in MATLAB.
\end{abstract}

Keywords: Attenuation, Electromagnetic, Line of Sight (LOS), Lookup angle, QPSK, Redundancy

\section{Introduction}

The purpose of this article is to come up with a computer model that places relays in positions that can guarantee full coverage of a predefined area. The system uses weight/cost between the relays to determine the communication link between the principal UAV and the control center. The system then applies the power budget to come up with the final path that is the relay UAVs that will take part in the actual exchange of information between the principal UAV and the control center.

For realistic communication between the control station and the autonomously flying aircraft we need to model for interfering factors that cause attenuation of the signal being transmitted. Due to factors as the Line of Sight, required data rates and so on, we need a set of UAVs performing shared tasks and at the same time maintaining the required inter vehicle actions to achieve communication between the command and the UAV.

Related work has been done in the field of coverage and measure of its effectiveness by the use of constructs such as the Voronoi diagram with other graph theoretical techniques (Merguerdichian, et al 2002, [1]). This enables the direct application of search techniques in the resulting topology representation from a team of UAVs. Also work has been done in the network architecture of UAVs. UAVs in a network can be placed in predetermined or stochastic configurations for any operation to take place. One such network is the close formation flying configuration (Bhargav, R Bellur et al 2000, [2]) where each UAV can communicate with its neighbors within the topology and by so doing can determine its neighbors.

1.1 Representation of the system

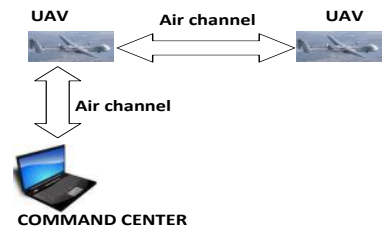

Fig. 1 Block diagram of how UAVs operate

From the command station the signal goes into the air communication channel. Here we need to model for interference. The signal then reaches the UAV, the UAV needs to be synchronized with carrier frequency of the antenna of the control station, hence we need to consider demodulation of the signal, measuring data rate being received.

\section{Proposed Solution}

This article proposes the solution that uses relays to provide a link between the autonomous UAV and the command center. The relays are in the form of other UAVs whose only function is to receive and forward 
data in either direction. In the forward direction we have communication from the command center to the UAV, and in the reverse direction communication is from the UAV to the command center. Information is therefore send via relays in either direction. The number of relays employed varies with the distance of separation between the principle UAV and the command center and also by the data rate expectations of the operation underway.

For successful communication we need to be sure that the whole area that we wish to fly our UAV is completely covered by the relays and the LOS can be achieved at any point on the network. The coverage should be $100 \%$ when the relays are placed in the topology.

\subsection{Achieving required coverage}

By hypothesis its required to determine the total number of observers necessary to cover an exhibition area such that every corner or every point of the exhibition area is monitored by at least one observer (Xiang Yang Li, et al 2003, [3]).if this is fulfilled then the exhibition area is said to be fully covered. In our case the exhibition area is the total area of interest and the observers being the relays. For instance given a predefined area as the dotted area on the diagram below and each relay covering a circular area:

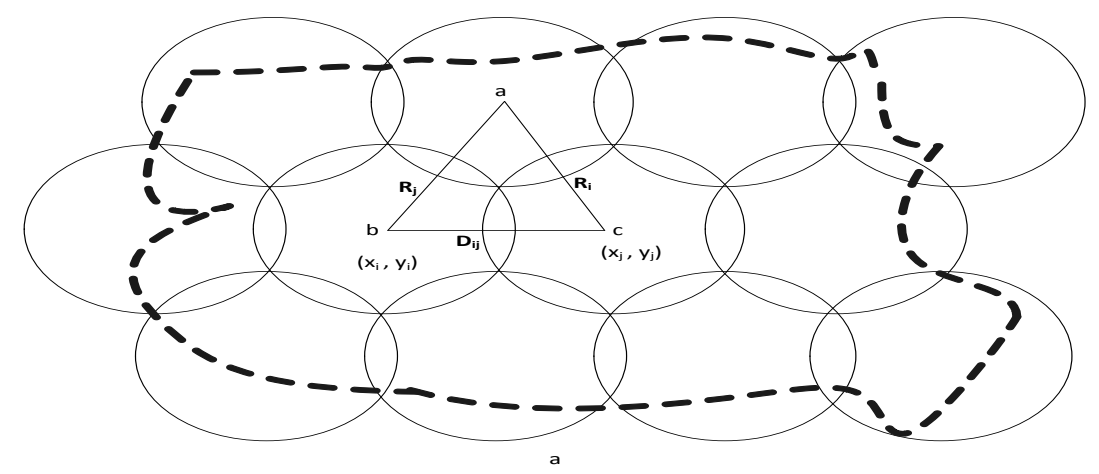

Fig. 2 how the relays should be deployed to have full coverage

To determine the position where each relay can be placed we use the calculations that follow. Using the triangle abc in Fig. 2 (M.P Singh et al 2000):

When $\mathrm{N}=$ set of relays in 2 Dimension

$$
\begin{aligned}
& \mathrm{N}=\left\{N_{1}, N_{2}, \ldots N_{i}\right\} \text { where } \mathrm{i}=\text { the total number of relays } \\
& R_{i}=\text { range one relay can cover given a } 10 \text { degree look up angle }
\end{aligned}
$$

$D_{i j}=$ distance between relay (i) and relay (j).

$D_{i j}=\sqrt{ }\left(\left|x_{i}-x_{j}\right|^{2}+\left|y_{i}-y_{j}\right|^{2}\right)$

Any point in the area ' $\mathrm{A}$ ' is said to be fully covered by the set $\mathrm{N}$, if the point is within reach of at least one relay $N_{i}$ for $i=1$ to the number of relays.

Mathematically an area A is fully covered if:

$$
\bigcup\left(\pi * R^{2}\right) \supset \mathrm{A}
$$

That is if the union of area of circle (circle represents area covered by one relay) is power set of specified area. Defining an area as a set of $\left(x_{i}, y_{i}\right)$ for $\mathrm{i}=1$ to the total number of relays.

In $r_{i}$ there is a set of coordinates $\left(x_{k}, y_{k}\right) \in\left\{x_{i}, y_{i}\right\}$ and an area is covered if

$$
\mathrm{A}=r_{1}\left(x_{k_{1}}, y_{k_{1}}\right) \cup r_{2}\left(, x_{k_{2}} y_{k_{2}}\right) \ldots \ldots \cup r_{n}\left(x_{k_{n}}, y_{k_{n}}\right)
$$

(1) and (2) when fulfilled, they define that every point is covered. Now if $D_{i j}<R_{i}+R_{j}$ then the neighbors (i) and (j) reach each other directly.

If $D_{i j}>R_{i}+R_{j}$ then $N_{i}$ and $N_{j}$ do not cover any arc of each other and there is not full coverage there.

\section{Design}

Depending on the distance of the UAV from the command, the signal send from the command may not reach it - because the earth is not flat and the signal maybe blocked by the curvature of the earth's surface as shown in Fig. 3. 
Given radius of the earth $=6300 \mathrm{~km}$ and lookup angle $=10^{\circ}$

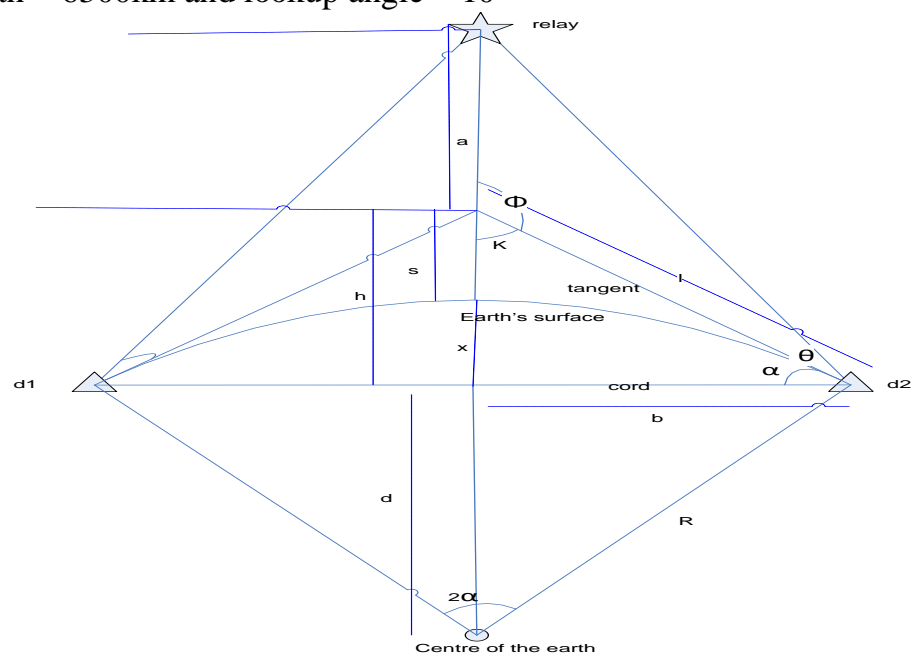

Fig 3. Illustration of the lookup angle and how the altitude of the UAV can be determined

The following defines the parameters used in Fig. 3. d1 is the starting point and $\mathrm{d} 2$ is the finishing point of the length of the whole area we need to be looking at.

$\mathrm{R}$ - is the radius of the earth

$\theta$ is the look up angle

' $A$ ' is the angle formed between the tangent and cord formed by the earth's surface of the area we need to look at for the design

' 1 ' is the length of the tangent from $\mathrm{d} 1$ or $\mathrm{d} 2$ to the vertical line from the relay to the earth's surface.

' $x$ 'is the distance from the earth's surface to the cord

' $s$ ' is the distance from the earth's surface to the point where the tangent intersects the vertical line.

' $h$ ' is the distance from the cord to the point of the same intersection as above.

' $d$ ' is the distance from the centre of the earth to the cord

' $b$ ' is the distance from $d 2$ to the vertical line

' $\alpha$ ' is the angle formed between the tangent and the cord.

$\Phi$ is the obtuse angle formed between the tangent and the vertical line

$\mathrm{d} 1=-460 \mathrm{~km}, \mathrm{~d} 2=460 \mathrm{~km}$ thus the relay covers the whole length of the predefined area which is approximately $920 \mathrm{~km}$ length.

\subsection{Calculations}

Arc length, $\mathrm{s}=2 * \alpha * \mathrm{R}$

$\alpha=920 / 6300=0.146$ radians, but 360 degrees $=2 \pi$ radians, hence $2 * \alpha=(0.146 / 2 \pi)^{*} 360$ degrees

And $\alpha=4.1835^{\circ}$

$\mathrm{d}=6300 * \cos (\alpha)=6284 \mathrm{~km}$

$\mathrm{b}=6300 * \sin (\alpha)=439 \mathrm{~km}$

$\mathrm{x}=\mathrm{R}-\mathrm{d}=16 \mathrm{~km}$

$\mathrm{h}=439 * \tan (\alpha)=30 \mathrm{~km}$ and $\mathrm{s}=14 \mathrm{~km}$.

Using the Pythagoras theorem $l^{2}=h^{2}+b^{2}=30^{2}+439^{2}$ which gives, $1=440 \mathrm{~km}$

Extracting the triangle with the angle, $\theta$ from Fig 4.

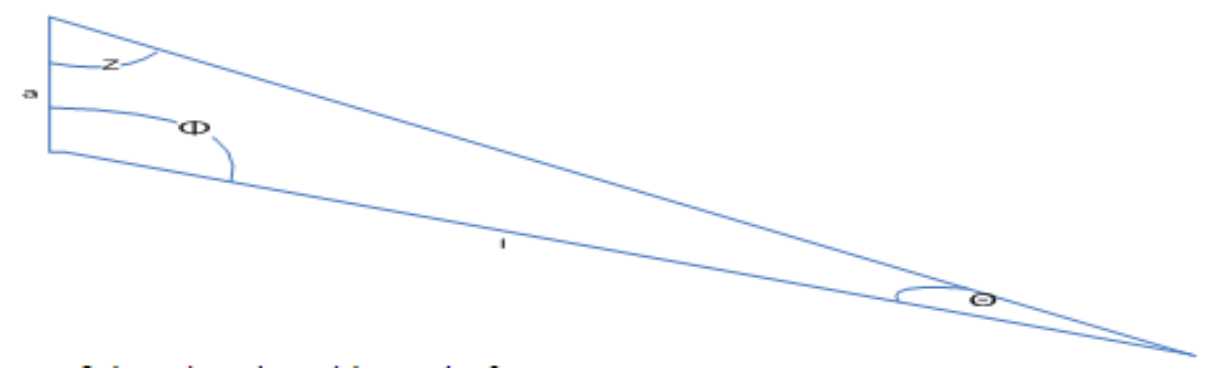

Fig 4.Extract of the triangle with angle $\theta$

For suitable line of sight then $\theta=10^{\circ}$, hence angle $\mathrm{K}$ is found from the right angled triangle which includes $\alpha$, and $K=86^{\circ}$. And hence $\Phi=180-86=94^{\circ}$ which gives angle $Z=76^{\circ}$ 
Applying the sine rule: $\frac{a}{\sin (10)}=\frac{440}{\sin (76)}$

This gives the distance, $\mathrm{a}=78 \mathrm{~km}$. Now the altitude $=\mathrm{a}+\mathrm{s}=78+14=92 \mathrm{~km}$ going up.

Conclusion- this distance is too high for any realistic operation to take place. Hence consider second option where we try to use two relays to provide for the LOS thus trying to lower h. Now consider the option of adding more relays to reduce altitude as shown in Fig. 5.

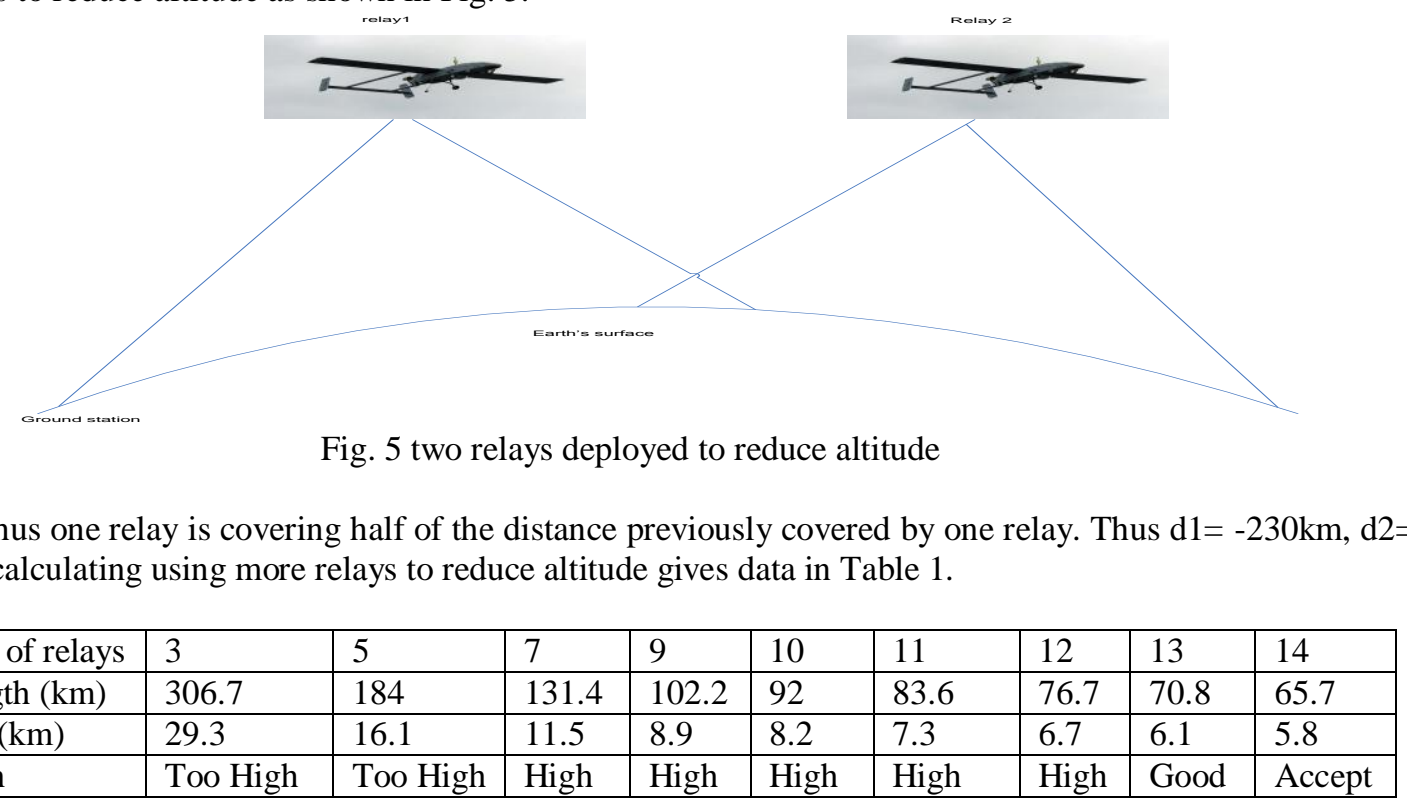

\subsection{Modelling the actual area}

At an altitude of $5.8 \mathrm{~km}$, one relay covers a circle of diameter $66 \mathrm{~km}$. Thus the placement of relays should be as shown in Fig. 6.

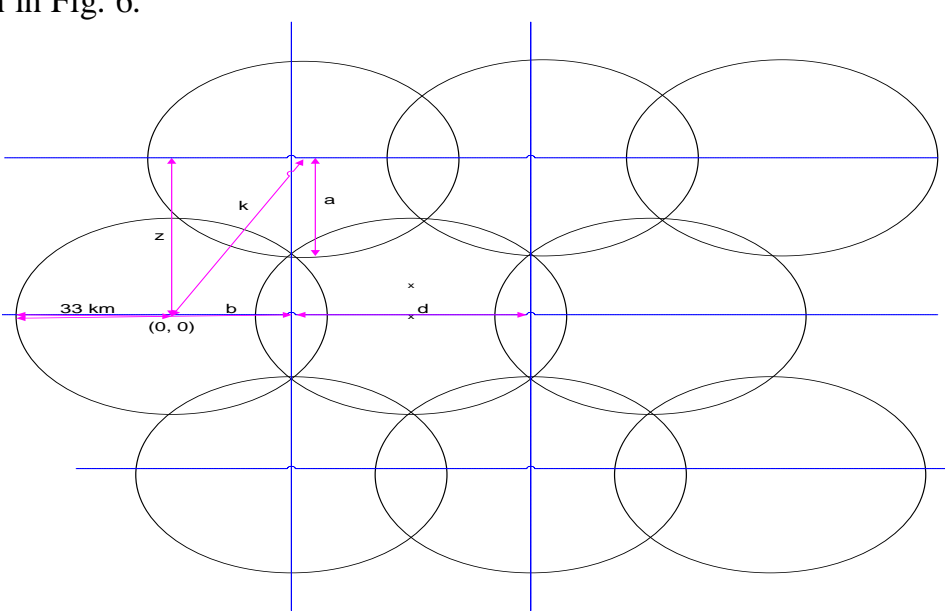

Fig. 6 positioning of relays for full coverage

When each relay overlaps to its horizontal neighboring relay by half of its radius we have $b=24.75 \mathrm{~km}, \mathrm{~d}=$ $49.5 \mathrm{~km}, \mathrm{a}=33 \mathrm{~km}, \mathrm{k}=60.16 \mathrm{~km}$ and $\mathrm{z}=54.83 \mathrm{~km}$

Consider a predefined area as shown in Fig. 7.

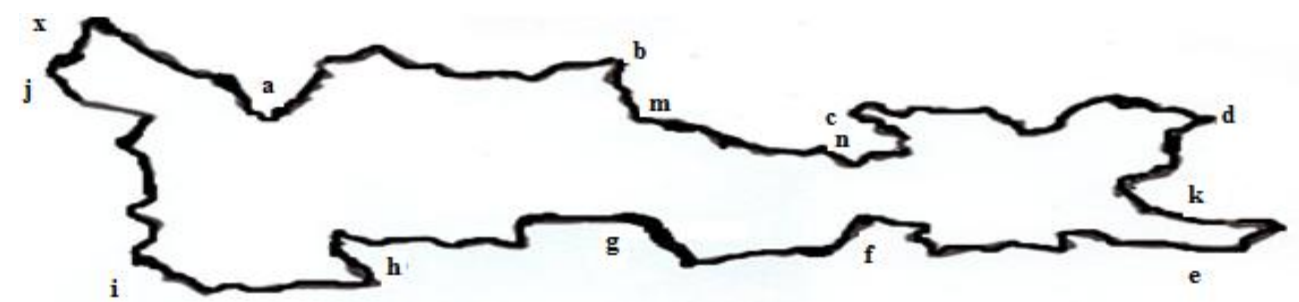

Fig. 7 the predefined area 
Approximate distances as labelled on the diagram being:

Table 2

\begin{tabular}{|l|l|l|l|l|l|l|l|l|l|l|l|l|l|l|l|}
\hline Points & j-a & a-b & m-c & c-d & c-n & n-f & d-k & k-e & x-i & b-m & i-h & h-g & g-f & f-e & j-d \\
\hline $\begin{array}{l}\text { Distance } \\
(\mathrm{km})\end{array}$ & 150 & 300 & 300 & 300 & 100 & 15 & 200 & 50 & 600 & 150 & 200 & 300 & 150 & 400 & 920 \\
\hline
\end{tabular}

For a predefined area as shown in Fig. 8, we need to place the relays so that they fulfill the principle of full coverage. For this article, a program was done in MATLAB to deploy the relays as shown in Fig. 8.

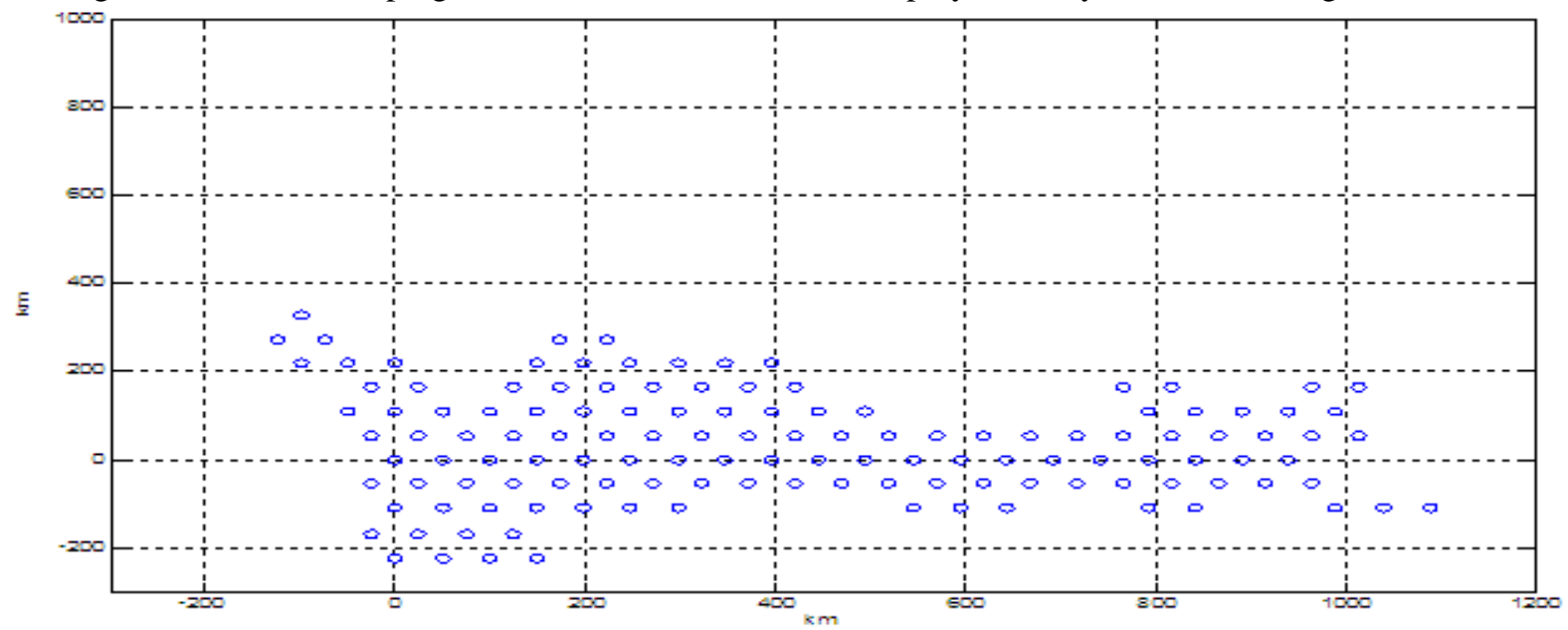

Fig. 8 actual modelled area deployed by relays

With the relays placed in full coverage positions, we need to establish a link that is a path which will be traversed by the data taking part in the communication process between the aircraft and the control station.

\subsection{The path}

With the given topology of deployed relays such as in Fig.9, we can construct several paths from the UAV to the command, hence we need to consider some optimization so that the communication is done using the best path. In this article we have used the Dijkstra's algorithm.

3.3.1 Dijkstra's Algorithm

Dijkstra's Algorithmis a graph search algorithm whose criteria is used in this design in order to come up with a desirable path. . For a given start relay on the topology the algorithm finds the path with the lowest cost/weight between that start relay and the command centre.

Consider Fig. 9

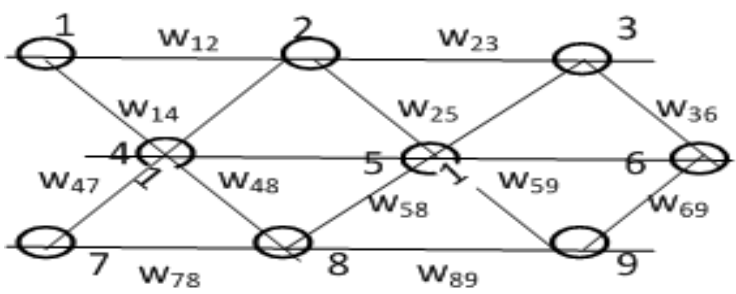

Fig. 9 relays in a topology

Where $\{1,2,3 \ldots 9\}$ are the relays, $\left\{\mathrm{W}_{12}, \mathrm{~W}_{23}, \ldots\right\}$ are the costs.

For example, if we start at relay 3 and we need to communicate with relay 7 there are so many nodes we can use to communicate. Each relay knows its neighbors, so it looks and compares the weights from it to each neighbor. It then chooses the neighbor with the smallest cost/weight as its next hop and the next hop will be in the direction of 7 . When at this next hop point, it repeats the same procedure until it reaches the command center. For example: from 3 we have neighbors with the weightsw $w_{23}, w_{36}$, and $w_{25}$ to consider. If $w_{23}$ is the smallest weight, then the next hop will be 2 , and at 2 the same procedure is followed until it reaches relay 7 .

3.4 Line of Sight restrictions

To set restrictions in the case when distance between the relays is too large.

The LOS equation (Eugeng Huang, et al 1998) can be stated as follows:

$\mathrm{R}=4.12 *\left(h^{1 / 2}+a^{1 / 2}\right)$

Where: $\mathrm{R}=$ range beyond which LOS is no longer available 


$$
\begin{aligned}
& \mathrm{h}=\text { height of one relay } \\
& \mathrm{a}=\text { height of the neighbor relay }
\end{aligned}
$$

In our case ' $\mathrm{h}$ ' and ' $\mathrm{a}$ ' are both the same and are $5000 \mathrm{~m}$ high for this calculation purpose.

Hence $\mathrm{R}=4.12 *\left(2 * 5000^{1 / 2}\right)=580 \mathrm{~km}$ apart. For the purpose of this article we set $\mathrm{R}=400 \mathrm{~km}$.

\section{Implementation}

Positions of relays are calculated according to the geometric calculations given in section 3.1. The values of the positions are stored in a matrix.

\subsection{Creation of connections between the relays}

We need to establish communication between each relay and it neighbors. To achieve this we use the function called DELAUNAY (Brian D. Hahn, Essential matlab for engineers and scientists,[4]). This function basically creates triangles for the given position of all the relays in the topology. For each instance it uses 3 relays and triangulates them. The triangles are such that their edges don't cross each other but an edge can be shared between two triangles and a relay can be encountered in more than one triangle. For example applying Delaunay to Fig. 8all the relays will be connected as illustrated in Fig. 10.

For each edge we need to determine its Euclidean distance and we get it by:

1: Get the $\left(x_{i}, y_{i}\right)$ coordinates of relay $i$ from the range matrix

2: Get the $\left(x_{j}, y_{j}\right)$ coordinates of relay $\mathrm{j}$ from the range matrix

Euclidian distance, $\left.\left.d=\sqrt{\left\{\left(x_{i}\right.\right.}-x_{j}\right)^{2}-\left(y_{i}-y_{j}\right)^{2}\right\}=$ distance between relay $\mathrm{i}$ and relay $\mathrm{j}$ which is the distance used to model the cost/weight between $\mathrm{i}$ and $\mathrm{j}$.

\subsection{Choosing the closest relay}

When the UAV is in the field, it automatically chooses the relay on the topology which is closest to it depending on the particular position it is operating at. To achieve this we make use of the function dsearch (William J Palm, Introduction to matlab for engineers, [5]). This function will acquire the position of the UAV, which can be got from GPS or other location discovery methods and then computes the location of the closest relay. Once we have the closest relay, we then need to get the path that will be traversed to exchange information.

\subsection{The path}

To get the path we need to determine the distance of each relay from the command center. In this article the command center first communicates with the relay at $(0,0)$ hence we will find the distance of each relay to this point.

Distance of relay (i), $D_{i}=\sqrt{ }\left(x_{i}^{2}+y_{i}^{2}\right)$ where $\mathrm{i}=1$ to the total number of relays.

The values of the distance of each relay from the command are stored in a matrix.

Now for the path:

1: At the start relay - determine its neighbors

2: If distance of any neighbor is greater than distance of the start relay to the command center then

remove this neighbor from the list

3: Apply the second constraint to the edges of the remaining neighbors.

- Get edge distances of each relay to the start relay

- Then near neighbor is the neighbor with the smallest edge distance among the potential neighbors to the start relay.

- With this near neighbor, we then apply the same procedure but this time starting at the near neighbor that is near neighbor becomes the start relay. 
Applying this procedure Fig. 10 is achieved with the relays showing the path to be traversed colored in green.

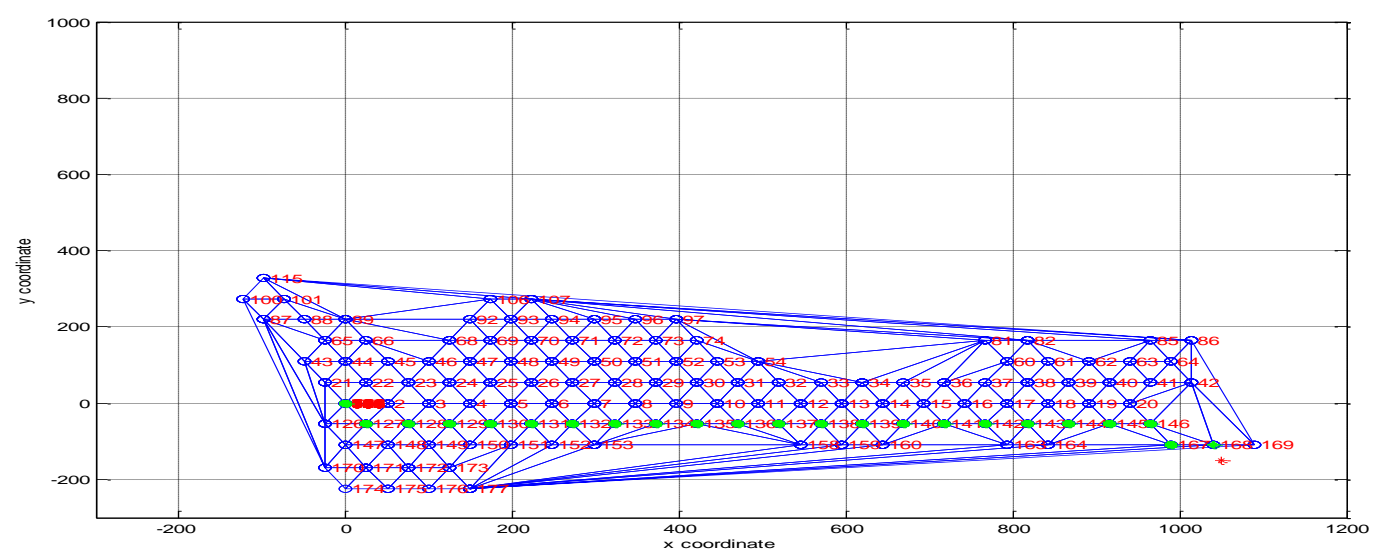

Fig. 10graph showing the path

Now when the path is identified we now need to determine the actual relays to take part in forming the link. This is achieved by calculations of the power budget. The parameters that we need to analyze are: (i) carrier frequency to be used for modulating the signal for transmission, (ii) transmit power used bythe system, (iii) the required data rate, (iv) LOS restrictions.

\subsection{Power Budget}

In this article QPSK (PSK with four states) modulation technique is proposed as it is able double the data rate as compared to BPSK while maintaining the same bandwidth. Also it can provide double data rate but probability of error being the same as BPSK and has less demand on linearity of the power amp.

\subsubsection{QPSK}

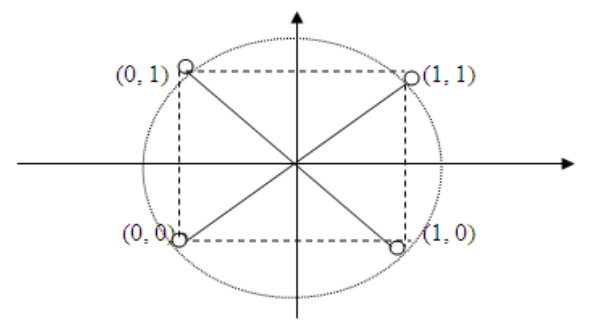

Fig. 11Constellation diagram

It is based on modulation of phase which is a version of frequency modulation where the phase of the carrier wave is modulated to encode bits of digital information in each phase change (MihajloStefanovic, et al 2003, [6])

To compare the data rate against distance for a certain communication of a specified central frequency and a particular power:

Assume the following values,

Temperature $=300 \mathrm{~K}$, Noise figure, $\mathrm{N}=2 \mathrm{~dB}$, Receiver gain $G_{r}=6 \mathrm{~dB}$, Transmitter gain $G_{t}=6 \mathrm{~dB}$, and the signal to noise ratio $=10 \mathrm{~dB}$.

We make use of the Friis equation which states that:

$P_{r}=P_{t} \cdot G_{t} \cdot G_{r} *\left\{\frac{\lambda}{4 \pi \mathrm{d}}\right\}^{2}$

Where $P_{r}$ is the power at the receiver.

$P_{t}=$ transmit power, $\lambda$ is the wavelength and ' $\mathrm{d}$ ' is the separation of two neighboring relays.

The noise is given as follows:

$P_{n}=\mathrm{k} * \mathrm{~T} * \mathrm{~N} * \mathrm{~B}$ 
Where $P_{n}$ is the noise power, T- Temperature, $\mathrm{N}$ is the noise figure, $\mathrm{B}$ is the bandwidth, and $\mathrm{k}$ is the Boltzmann constant, $1.38 * 10^{-23}$

Signal to noise ratio, $\mathrm{SNR}=\frac{P_{r}}{P_{n}}$

For free space the losses, L, are given by:

$\mathrm{L}=\left\{\frac{4 \pi d}{\lambda}\right\}^{2}$

Expressed in $\mathrm{dB}$ we have

$\mathrm{L}(\mathrm{dB})=32+20 * \log \left(d_{k m}\right)+20 * \log \left(f_{M H Z}\right)$

Where $-\mathrm{d}$ is the separation distance of the relays in $\mathrm{km}$.

$-\mathrm{f}$ is the central frequency of operation in $\mathrm{MHz}$.

Now using this equation and assuming other losses due to precipitation, humidity, electromagnetic losses and such others that are accounted by another $10 \mathrm{~dB}$ we get results as shown in Table 3

Table 3

\begin{tabular}{|l|l|l|l|l|l|l|}
\hline Distance $(\mathrm{km})$ & 50 & 100 & 150 & 200 & 300 & 400 \\
\hline Total losses $\mathrm{L}_{\mathrm{t}}$ for $500 \mathrm{MHz}$ & 130 & 136 & 139 & 142 & 145 & 148 \\
\hline Total losses $\mathrm{L}_{\mathrm{t}}$ for $900 \mathrm{MHz}$ & 135 & 141 & 144 & 147 & 150 & 153 \\
\hline Total losses $\mathrm{L}_{\mathrm{t}}$ for $1.4 \mathrm{GHz}$ & 139 & 145 & 148 & 151 & 154 & 157 \\
\hline
\end{tabular}

We the use these losses to determine the EIRP - effective isotropic radiated power at certain transmit powers and different frequencies.

$\mathrm{EIRP}=P_{t} * G_{t}$

EIRP expressed in dB, EIRP $(\mathrm{dB})=10 * \log \left(P_{t}\right)+10 * \log \left(G_{t}\right)$

But $G_{t}=4=6 \mathrm{~dB}$

For $P_{t}=1 \mathrm{~mW}=-30 \mathrm{~dB}$ we have EIRP $(\mathrm{dB})=-30+6=-24 \mathrm{~dB}$

For $10 \mathrm{~mW}=-20 \mathrm{~dB}$ we have $\operatorname{EIRP}(\mathrm{dB})=-20+6=-14 \mathrm{~dB}$

For $100 \mathrm{~mW}=-10 \mathrm{~dB}$ we have EIRP $(\mathrm{dB})=-10+6=-4 \mathrm{~dB}$

For $1 \mathrm{~W}=0 \mathrm{~dB}$ we have EIRP $(\mathrm{dB})=6 \mathrm{~dB}$

Now for realistic communication we need to maintain a signal to noise ratio (SNR) of around $10 \mathrm{~dB}$. Applying (5) for SNR we have:

$\mathrm{SNR}=\frac{P_{r}}{P_{n}}$

And using (3) and (4) given above for $P_{r}$ and $P_{n}$ we progress as follows:

$\mathrm{SNR}=\frac{\mathbf{P}_{\mathrm{t}} * \mathrm{G}_{t} * \mathrm{G}_{r}}{\boldsymbol{k} * \boldsymbol{T} * \boldsymbol{N} * \boldsymbol{B}}\left\{\frac{\lambda}{4 \pi d}\right\}^{2}$

Expressed in $\mathrm{dB}$, we have:

$\mathrm{SNR}(\mathrm{dB})=\mathrm{EIRP}-L_{t}+10 * \log \left(G_{r}\right)-10 * \log (\mathrm{T})-10 * \log (\mathrm{k})-10 * \log (\mathrm{B})+\mathrm{N}$

(11)

Substituting the actual values into equation (11): $\mathrm{T}=300 \mathrm{~K}, \mathrm{k}=1.38 * 10^{\wedge}-23, \mathrm{~N}=2 \mathrm{~dB}$ and $\mathrm{SNR}=10 \mathrm{~dB}$

$10=\mathrm{EIRP}-L_{t}+6-10 * \log (300)-10 * \log \left(1.38 * 10^{\wedge}-23\right)-10 * \log (\mathrm{B})+2$

Simplifies to:

$10=\mathrm{EIRP}-L_{t}+6-24.8-228.6-10 * \log (\mathrm{B})+2$ this leads to:

$-201=\mathrm{EIRP}-L_{t}-10 * \log (\mathrm{B})$

And we rearrange to formulate a new equation as follows:

$10 * \log (\mathrm{B})=\mathrm{EIRP}-L_{t}+201$ 
4.4.2 Determining Data Rate (bps)

Applying (12) and substituting with appropriate parameters we get results as shown in Table 4:

Table 4

\begin{tabular}{|l|l|l|l|l|l|l|}
\hline Distance $(\mathrm{km})$ & 50 & 100 & 150 & 200 & 300 & 400 \\
\hline Data rate for $1 \mathrm{~mW}$ & $10^{4.7}$ & $10^{4.1}$ & $10^{3.8}$ & $10^{3.5}$ & $10^{3.2}$ & $10^{2.9}$ \\
\hline Data rate for $10 \mathrm{~mW}$ & $10^{5.7}$ & $10^{5.1}$ & $10^{4.8}$ & $10^{4.5}$ & $10^{4.2}$ & $10^{3.9}$ \\
\hline Data rate for $100 \mathrm{~mW}$ & $10^{6.7}$ & $10^{6.1}$ & $10^{5.8}$ & $10^{5.5}$ & $10^{5.2}$ & $10^{4.9}$ \\
\hline Data rate for $1 \mathrm{~W}$ & $10^{7.7}$ & $10^{7.1}$ & $10^{6.8}$ & $10^{6.5}$ & $10^{6.2}$ & $10^{5.9}$ \\
\hline
\end{tabular}

Using these calculations we can draw a graph, Fig. 12, of variation of bit rate with distance for a given amount of transmit power as follows.

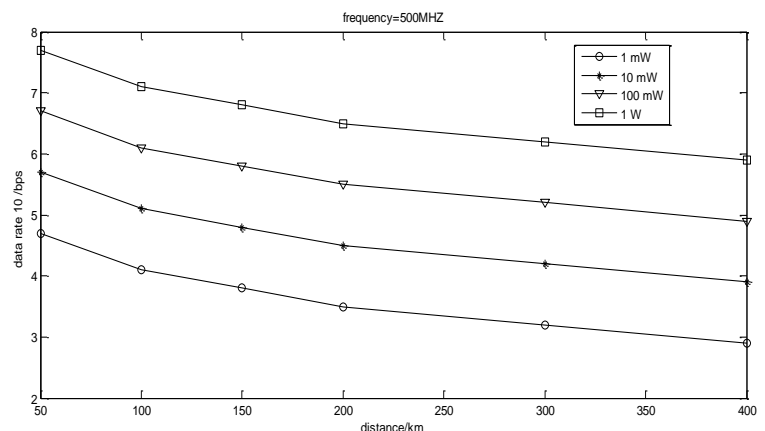

Fig. 12 variation of bandwidth with distance for $500 \mathrm{MHz}$

For $900 \mathrm{MHz}$ and $1400 \mathrm{MHz}$ we can perform the same calculations and have the information of variation of bit rate with distance at given transmit powers in the same manner we did for the $500 \mathrm{MHz}$.

For $900 \mathrm{MHz}$

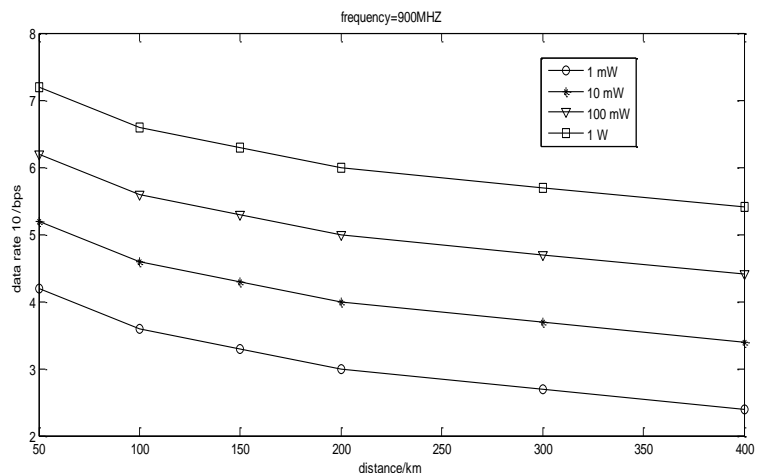

For $1.4 \mathrm{GHz}$

Fig. 13 variation of bandwidth with distance for $900 \mathrm{MHz}$

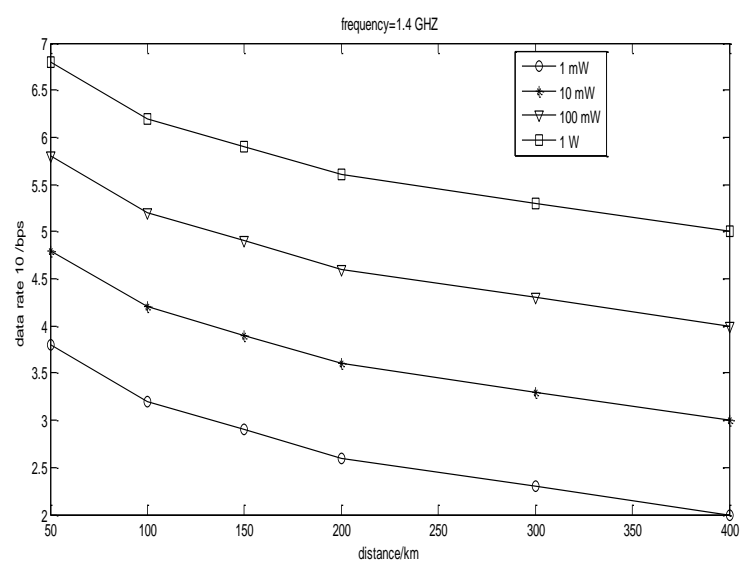

Fig. 14variation of bandwidth with distance for $1.4 \mathrm{GHz}$ 


\section{Results}

When the program is executed, on the screen we have the relays plotted in a way that they provide full coverage for the whole predefined area. The numbers are the identities of each relay. Each position for the relays is stored in matrix. On the command screen of the program, it prompts the user to enter the position of the UAV (the aircraft we want to control and command). The user is required to enter the $\mathrm{x}$ coordinate and then the $\mathrm{y}$ coordinate. This position of the UAV is then plotted on the graph and is indicated by a red star on the screen. From there it goes straight to determine the closet relay to it, and then a possible path in turn is calculated. This is the best path formed from the first relay to the command center given that particular topology of relays. If topology changes the path changes too.

\subsection{Tests}

\subsubsection{Change of position for the UAV}

For $\mathrm{x}=1050$ and $\mathrm{y}=-150$

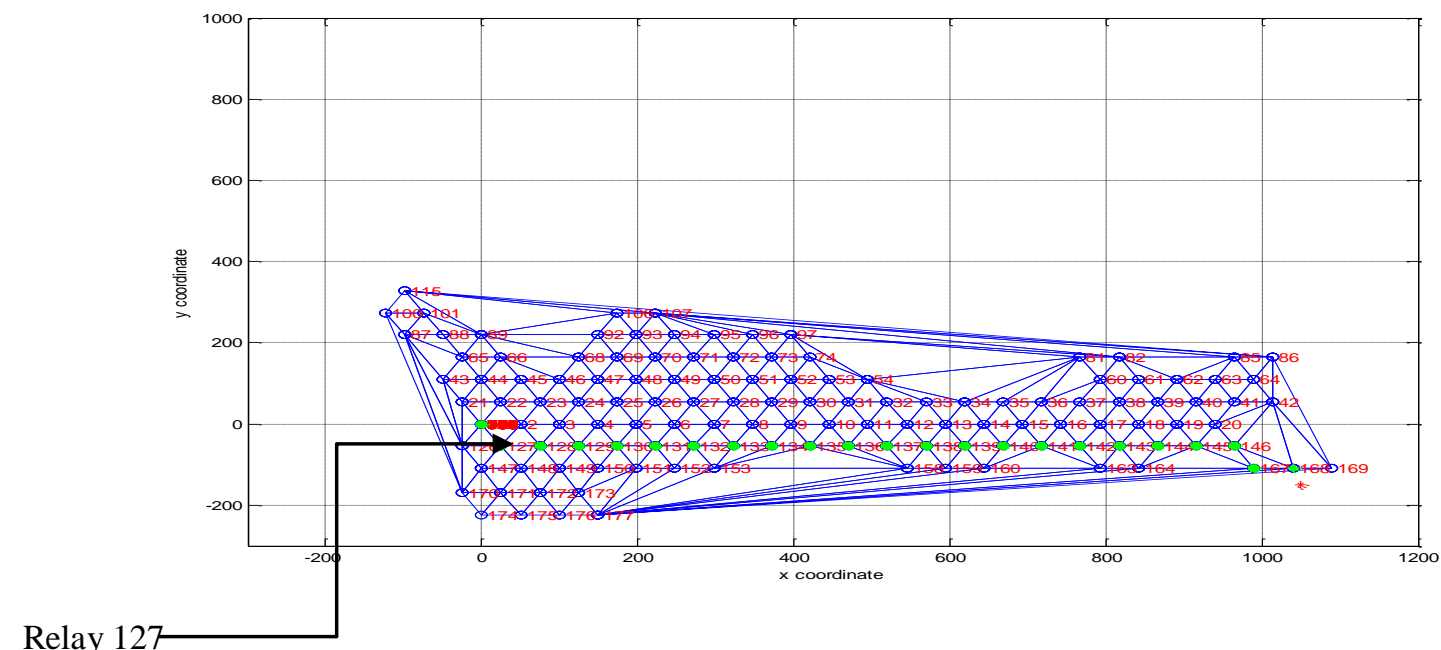

Fig. 15path formed for $\mathrm{x}$ and $\mathrm{y}$ coordinates of 1050 and $-150 \mathrm{~km}$ respectively.

For $\mathrm{x}=450, \mathrm{y}=220$

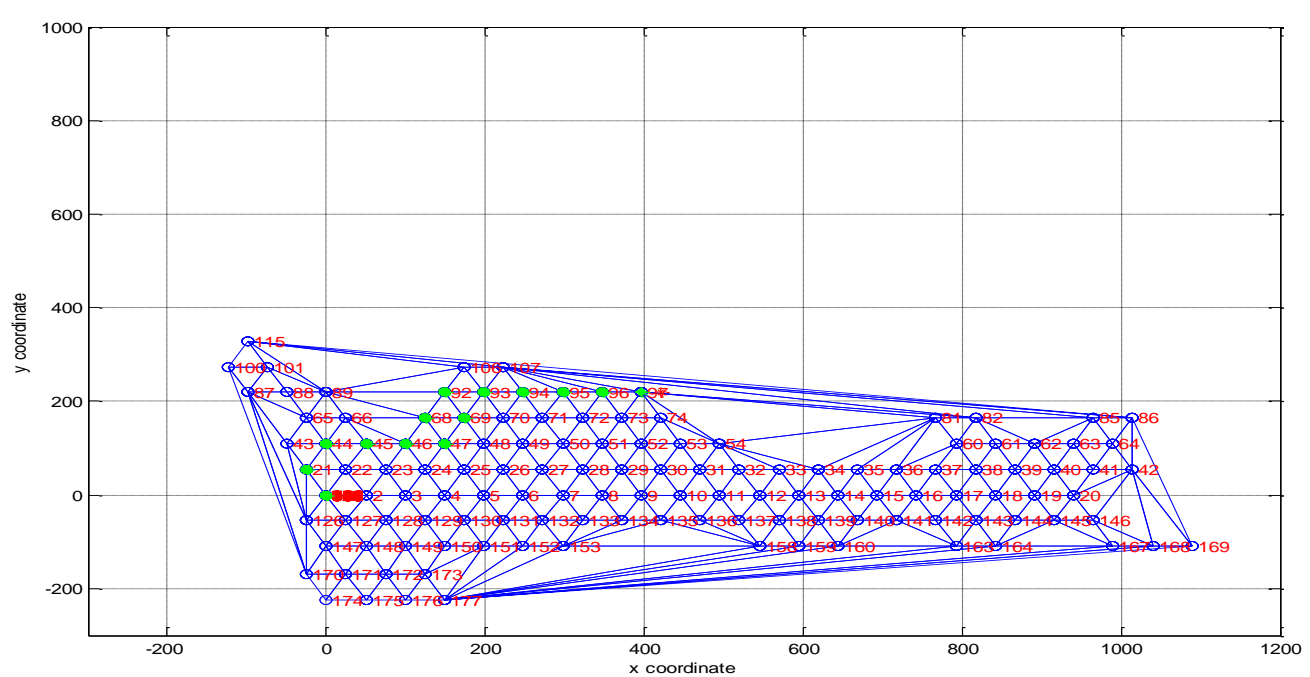

Fig. 16path formed for $\mathrm{x}$ and $\mathrm{y}$ coordinates of 450 and $220 \mathrm{~km}$ respectively.

\subsubsection{Change of topology}

Assume relays 126, 127, 138 which were part of the path in condition 1 above when $\mathrm{x}=1050, \mathrm{y}=-150$.

The path formed now is as follows: 


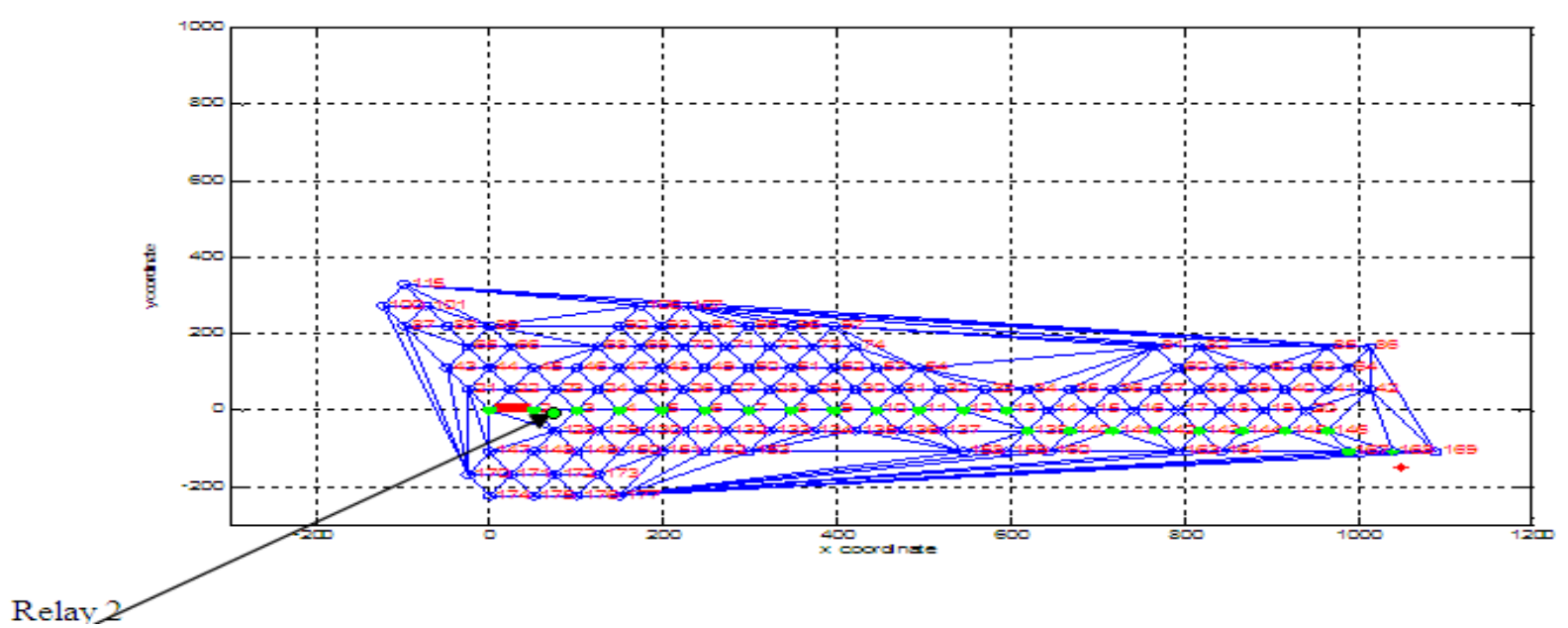

Fig. 17Path formed for $\mathrm{x}$ and $\mathrm{y}$ coordinates of 1050 and $-150 \mathrm{~km}$ with relays 126,127 and 138 removed.

Here relay 2 is the one now communicating with the command, but before the change relay 127 was the one communicating with the command as shown in Fig. 15.

\subsection{Applying the power budget}

Now with this path, we need to apply some optimization, consider different data rates, different transmit powers, different carrier frequencies and so depending on the parameters chosen. On the command screen a prompt that says enter power is shown. Another prompt for frequency appears. And at last a prompt for required bit rate comes on. If the parameters entered are practical, the program then shows the relays from the path which will take part in final communication from the UAV to the command center.

These relays are in blue color. And after this the graph will bear the title which states the parameters that were used. When any of the parameters are changed the path changes accordingly. If no relays appear in blue, then it means the parameters selected are not fulfilling some set conditions and on the command screen it outputs a statement which says what the problem is.

In summary the blue relays form the path which will be used for the actual communication process at the stated parameters.

\subsubsection{Examples}

When the program is run, it prompts the user to enter the position of the UAV.

Then the screen prompts:

\{ Frequency values choose either $500,900,1.4 \mathrm{GHz}$

Power choose either $1,0.1,0.01,0.001 \mathrm{~W}$

Enter data rate as $10^{\wedge}$ interger

Enter Power......

This gives guidelines for the parameters which system was designed for: For parameters: frequency $=$ $900 \mathrm{MHz}$, power $=10 \mathrm{~mW}$ and data rate $=10^{\wedge} 3, \mathrm{x}$ and y coordinates being 820 and $-180 \mathrm{~km}$ respectively we have:

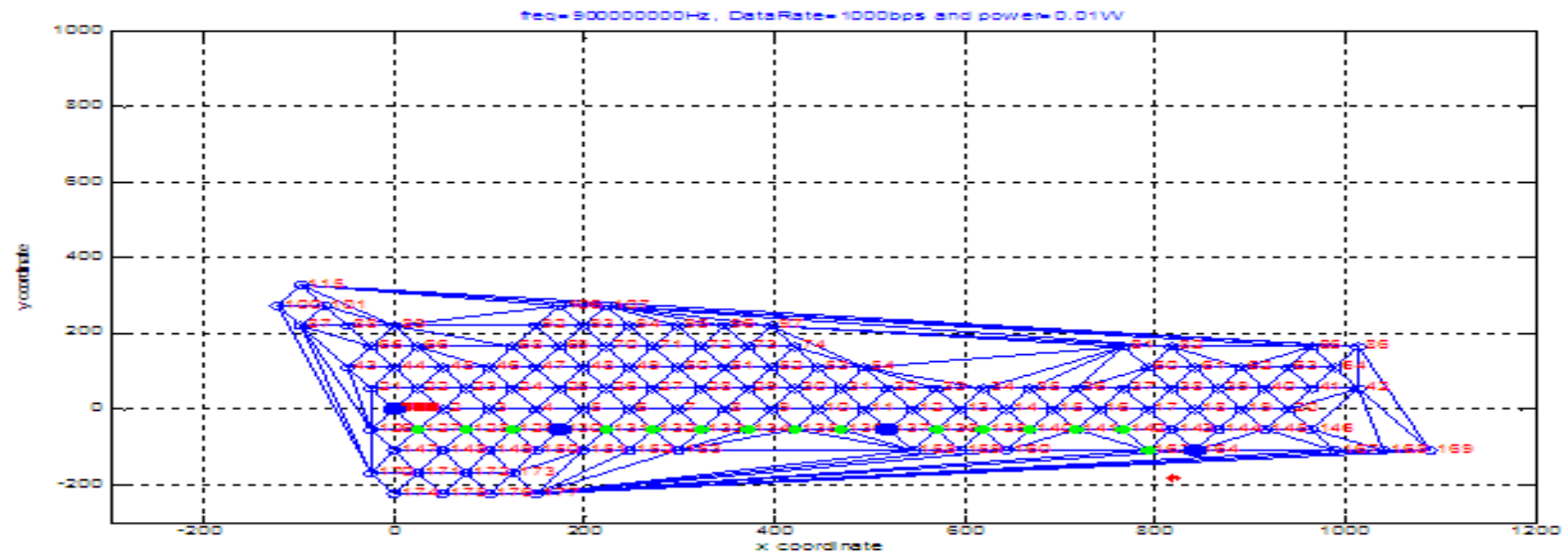

Fig. 18 blue relays forming communication path for frequency $=900 \mathrm{MHz}$, power $=10 \mathrm{~mW}$, data rate $=10^{\wedge} 3 \mathrm{bps}$ for coordinates $\mathrm{x}=820 \mathrm{~km}$ and $\mathrm{y}=180 \mathrm{~km}$ 
For the same parameters as above but a data rate of $10^{\wedge} 5$ we have:

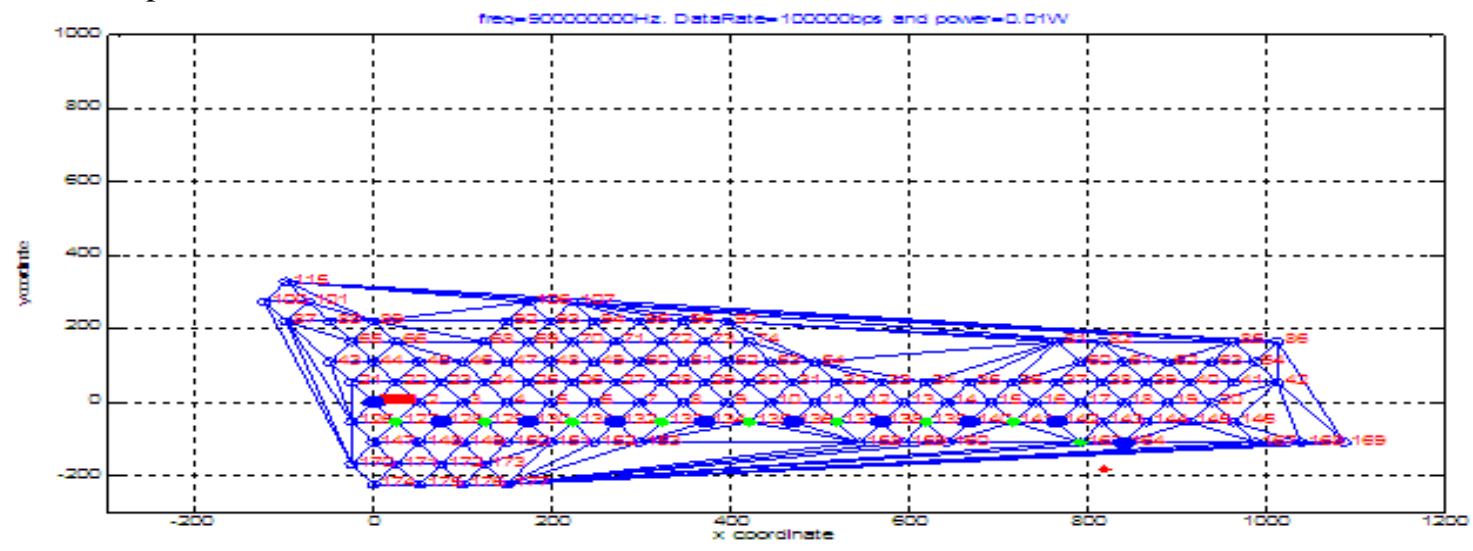

Fig. 19 communication path when data rate is $10^{\wedge} 5 \mathrm{bps}$

For a frequency of $1.4 \mathrm{GHz}$, power $=1 \mathrm{~W}$, data rate $=10^{\wedge} 4, \mathrm{x}=1010, \mathrm{y}=199$ we have:

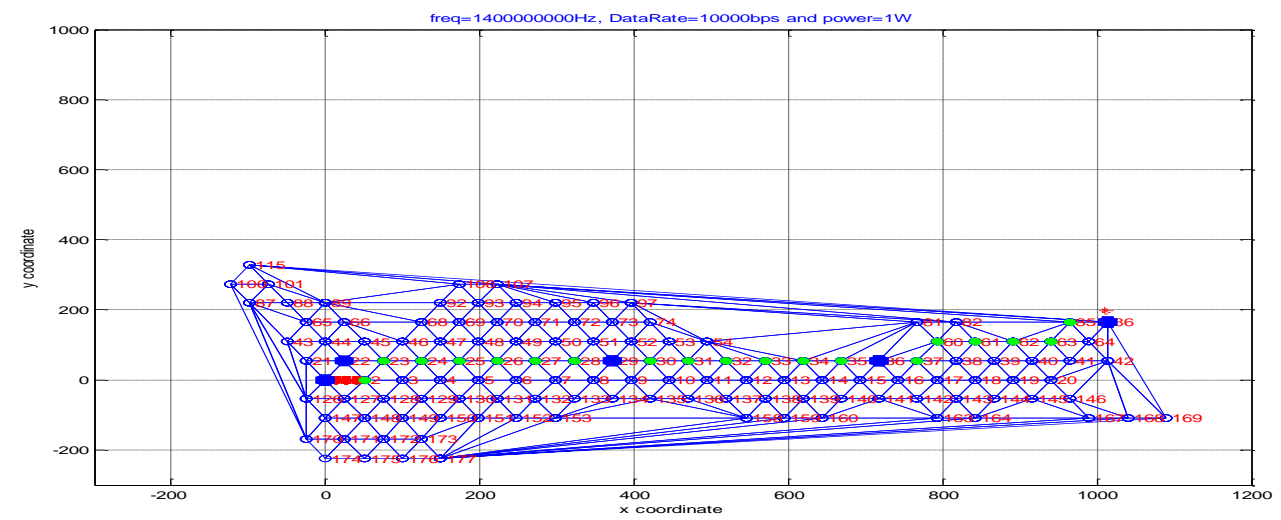

Fig. 20 blue relays forming communication path for frequency $=1.4 \mathrm{GHz}$, power $=1 \mathrm{~W}$, data rate $=10^{\wedge} 4 \mathrm{bps}$ for coordinates $\mathrm{x}=1010 \mathrm{~km}$ and $\mathrm{y}=199 \mathrm{~km}$

Changing only the transmit power from the above to $100 \mathrm{~mW}$ we get:

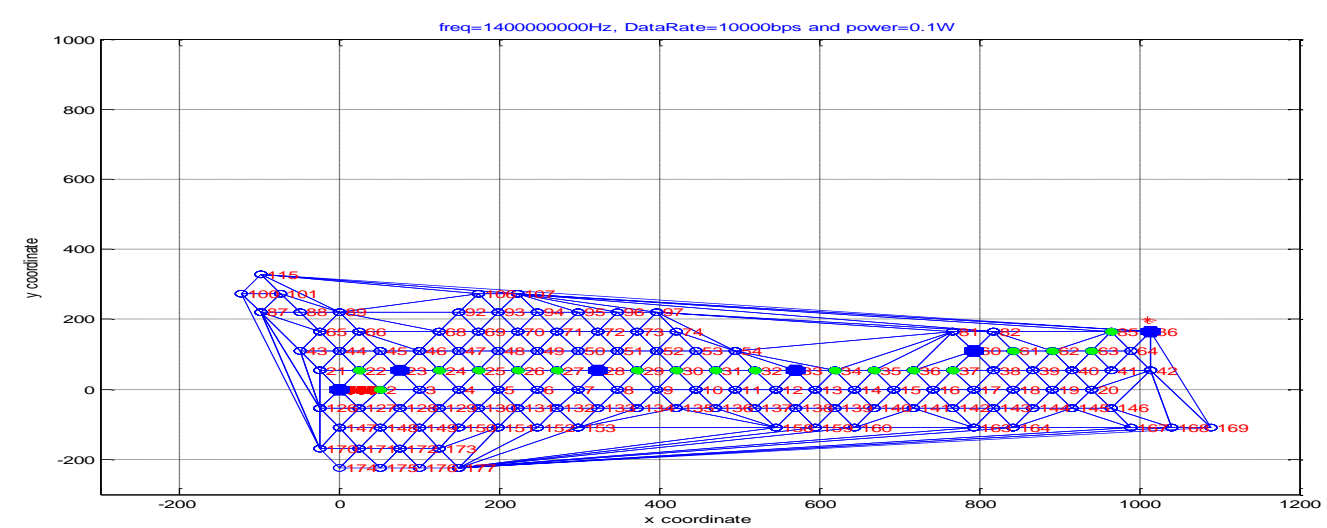

Fig. 21 communication path when power $=100 \mathrm{~mW}$

\subsection{Line of Sight restrictions}

For certain parameters it can be noted from the power budget calculations that we can go for larger distances of more than $500 \mathrm{~km}$ but still getting the required bit rate which implies it can be possible for us to use relays that are $500 \mathrm{~km}$ apart. But due to the Line Of Sight restrictions, this distance is not achievable.

Example 1: frequency $=500 \mathrm{Mhz}$, power $=1 \mathrm{~W}$, data rate $=10^{\wedge} 5$, and $\mathrm{x}$ and y coordinates $=1010,180$ respectively we get: 


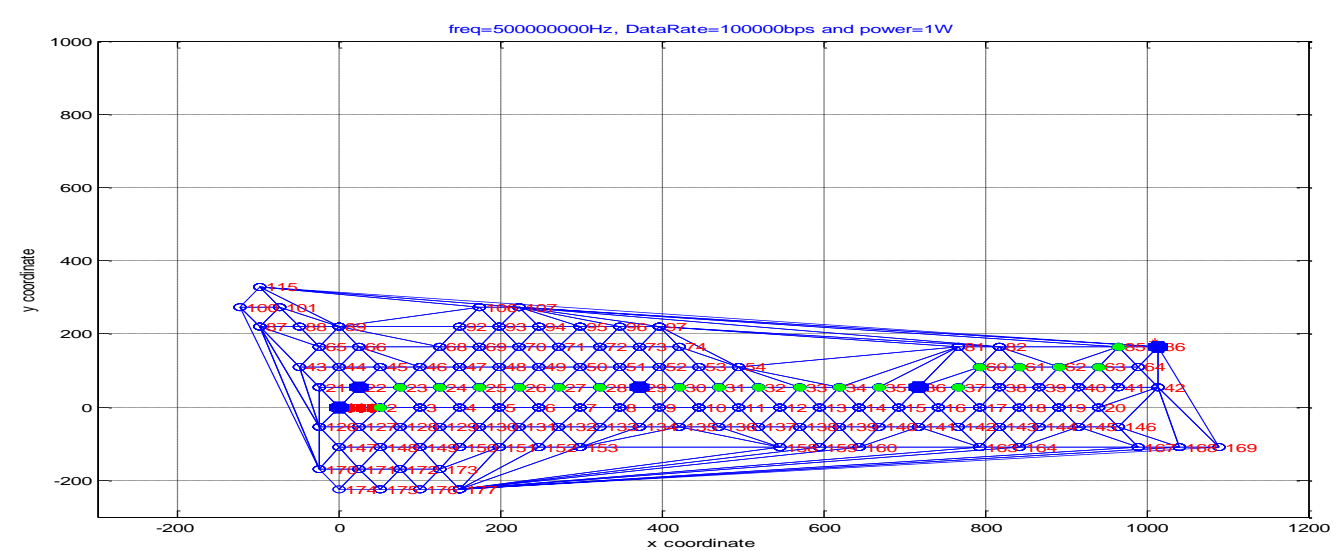

Fig. $22 \mathrm{blue}$ relays forming communication path for frequency $=500 \mathrm{MHz}$, power $=1 \mathrm{~W}$, data rate $=10^{\wedge} 5 \mathrm{bps}$ for coordinates $\mathrm{x}=1010 \mathrm{~km}$ and $\mathrm{y}=180 \mathrm{~km}$

We notice from Fig. 13 that with the stated power, distances greater than $400 \mathrm{~km}$ can be achieved but here due to LOS restrictions the maximum distance between the relays forming the final link is limited to about $400 \mathrm{~km}$.

\subsection{Entry of incorrect parameters}

On coordinates, if a character is entered instead of an integer, the program keeps prompting the user to enter the coordinates. If one or more parameters of frequency, power or so are incorrectly entered the program reports an error message and the user will have chance to restart entering the parameters.

When the data rate is not achievable for a specified power or set of parameters, the program will output an error message, "data rate not reachable"

\section{Conclusions}

This solution of employing relay UAVs has the major strength of being very robust. It can be shown on the system that a loss of several relay UAVs in a topology doesn't affect communication at all. It can be realized also that it's even more robust when the system employs a lower central frequency, a higher transmit power and requires a lower bit rate. However the limitation of the system stands on the fact that the relays considered are static, hence the authors will continue with further studies on the same issue where mobile relay UAVs would be employed and work on variable altitude terrain. The system can find use in military operations, agriculture, civil operations among other things for planning UAV system architectures and investments.

\section{Journal Papers:}

\section{REFERENCES:}

[1] Meguerdichian, S., Koushanfar, F. ,Potkonjak,M.and Srivastava, M.B, Coverage problems in wireless ad-hoc sensor networks,IEEE Computer and Communications Societies. Proceedings, vol 3, 2001.

[2] Bhargav R. Bellur, Mark G. Lewis and Fred L. Templin, An Ad-hoc Network for Teams of Autonomous Vehicles, SRI International, 2000

[3] Xiang- Yang Li, Peng- Ju Wan and OphirFrieder, Coverage in wireless ad- hoc sensor networks, IEEE transactions on communications, vol 3, 2001.

\section{Books:}

[4] Brian D. Hahn, D.T Valentine, Essential Matlab for engineers and scientists (Butterworth-Heinemann Inc, 2007)

[5] William J Palm, Intoduction to matlab for engineers (University of Rhode Island, third edition)

[6] Mihajlo C. Stefanovic ${ }^{*}$, Goran T. Djordjevic, BPSK and QPSK non-linear satellite communication system performance in the presence of cochannel interference, International Journal of Satellite Communications and Networking Volume 21, Issue 3, pages 285-297, May/June 2003 